\title{
Quantification of MYCN, DDX1, and NAG Gene Copy Number in Neuroblastoma Using a Real-Time Quantitative PCR Assay
}

\author{
Katleen De Preter, M.Sc.,Frank Speleman, Ph.S.Valérie Combaret, Ph.D., John Lunec, Ph.D., \\ Geneviève Laureys, M.D., Ph.D., Bert H.J. Eussen, Nadine Francotte, M.D., \\ Julian Board, Andy D.J. Pearson, M.D., Anne De Paepe, M.D., Ph.D., Nadine Van Roy, Ph.D., \\ Jo Vandesompele, M.Sc. \\ Center for Medical Genetics (KDP, FS, ADP, NVR, JV), and Department of Pediatric Hemato-Oncology \\ (GL), Ghent University Hospital, Ghent, Belgium; Molecular Oncology Unit (VC), Centre Léon Bérard, \\ Lyon, France; Cancer Research Unit (JL, JB), Department of Child Health (AP), University of Newcastle, \\ Newcastle upon Tyne, United Kingdom; Department of Clinical Genetics (BE), Erasmus University, \\ Rotterdam, The Netherlands; and Department of Pediatrics (Hemato-Oncology section) (NF), Cliniques \\ Saint-Joseph Espérance, Montegnée, Belgium
}

\begin{abstract}
Amplification of the proto-oncogene $M Y C N$ is a strong adverse prognostic factor in neuroblastoma patients in all tumor stages. The status of the $M Y C N$ gene has become an important factor in clinical decision making and therapy stratification. Consequently, fast and accurate assessment of MYCN gene copy number is of the utmost importance and the use of two independent methods to determine MYCN status is recommended. For these reasons we have developed and evaluated a real-time quantitative PCR (Q-PCR) assay as an alternative for timeconsuming Southern blot analysis (SB), and as a second independent technique in parallel with fluorescence in situ hybridization (FISH) analysis. Advantages of Q-PCR are a large dynamic range of quantification, no requirement for post-PCR sample handling and the need for very small amounts of starting material. The accuracy of the assay was illustrated by measurement of $\mathrm{MYCN}$ single gene copy changes in DNA samples of two patients with 2p deletion and duplication, respectively. Two different detection chemistries i.e., a sequence specific TaqMan probe and a generic DNA binding dye SYBR Green I were evaluated and shown to yield similar
\end{abstract}

Copyright (C) 2002 by The United States and Canadian Academy of Pathology, Inc.

VOL. 15, NO. 2, P. 159, 2002 Printed in the U.S.A

Date of acceptance: December 11, 2001.

Katleen De Preter is an aspirant with the Fund for Scientific Research, Flanders (FWO-Vlaanderen). Nadine Van Roy is a postdoctoral researcher with the FWO. The work was also supported by the Flemish Institute for the Promotion of Scientific Technological Research in Industry (IWT), BOF-grant 011F1200 and 011B4300, GOA-grant 12051397 and FWO-grant G.0028.00.

Address reprint requests to: Frank Speleman, Center for Medical Genetics, Ghent University Hospital 1K5, De Pintelaan 185, 9000 Ghent, Belgium; e-mail: franki.speleman@rug.ac.be; fax: 32-(0)9-2404970. results. Also, two different calculation methods for copy number determination were used i.e., the kinetic method and the comparative $\mathrm{C}_{\mathrm{T}}$ method, and shown to be equivalent. In total, 175 neuroblastoma samples with known MYCN status, as determined by FISH and/or SB, were examined. Q-PCR data were highly concordant with FISH and SB data. In addition to $M Y C N$ copy number evaluation, $D D X 1$ and $N A G$ gene copy numbers were determined using a similar Q-PCR strategy. Survival analysis pointed out that $D D X 1$ and/or $N A G$ amplification has no additional adverse effect on prognosis.

KEY WORDS: DDX1 amplification, MYCN amplification, $N A G$ amplification, Neuroblastoma, Real-time quantitative PCR, SYBR Green I, Survival.

Mod Pathol 2002;15(2):159-166

Neuroblastoma (NB) is the most frequent extracranial solid tumor in children below the age of 5 years. NB shows a wide clinical and genetic heterogeneity (1): most patients with localized disease (stages 1 and 2) are infants (less than 1 year) with excellent survival after surgical treatment only, whereas most older children ( $\geq 1$ year) have widespread metastasis (stages 3 and 4 ) and need additional chemotherapy and hematopoietic stem cell rescue. Stage 4S tumors predominantly occur in infants and may regress and disappear without treatment. Age and clinical stage are two important clinical parameters in prediction of treatment outcome and survival. In addition, many biological parameters have been tested for their prognostic power to further improve the selection of patients, which require intensive 
treatment (2). Amplification of the MYCN protooncogene was recognized as a strong independent adverse prognostic factor (in particular in patients with 1, 2, and $4 \mathrm{~S}$ disease stage) (3) and has been widely used in treatment stratification. More recently, $17 \mathrm{q}$ gain was shown to be the most powerful predictive factor and for this reason $17 \mathrm{q}$ status is also being increasingly analyzed in clinical samples (4).

For detection of MYCN amplification (MNA) both fluorescence in situ hybridization (FISH) and Southern blot analysis (SB) have been applied. FISH allows rapid and accurate determination of MYCN copy number, allows MYCN copy number evaluation at the single cell level (5) and can be performed on tumor imprints of biopsies that can be evaluated for tumor cell morphology and content by combination with immunohistochemical staining (6). Despite the high sensitivity and specificity of FISH for detection of MNA, errors in assessment of $M Y C N$ copy number, albeit rare, may occur. Consequently application of two independent methods for analysis of $M Y C N$ gene copy number has been proposed (Ambros et al., submitted).

In most laboratories SB was the standard method for the assessment of MYCN gene copy number. Unfortunately, SB is laborious, time-consuming and requires considerable amounts of DNA from fresh or frozen samples. To circumvent these problems a competitive or semi-quantitative PCR assay has been introduced $(7,8)$, but both methods offer only semi-quantitative analysis of gene amplification in a small dynamic range and are prone to cross contamination due to post-PCR handling.

These problems were recently overcome by the introduction of real-time quantitative PCR (Q-PCR). This technique offers major advantages compared with conventional methods and former PCR based strategies, including the large dynamic range of quantification, the exclusion of post-PCR manipulations, thus greatly reducing the risk of carry-over contamination, the possibility to perform the assay on only minimal amounts of tumor material and the high-throughput capacity $(9,10)$.

The major aim of this study was to design and validate a Q-PCR assay for fast and accurate detection of MNA in surgical NB samples. To this purpose, MYCN copy numbers were determined in 141 primary NB tumors, 34 NB cell lines, two patients with a constitutional deletion or duplication involving the MYCN locus, and normal leukocyte DNA samples. These results were compared with previous FISH and/or SB results. In addition we determined the copy number of DDX1 and NAG. Both genes are known to be co-amplified in subsets of MYCN amplified tumors (11-14). The copy numbers of the two latter genes were used to determine a possible correlation of co-amplification with survival and risk to relapse of the patient.

\section{MATERIALS AND METHODS}

\section{Tumor Samples and DNA Isolation}

NB tumor samples were collected at the Ghent University Hospital (Ghent, Belgium) ( $n$-20), in the Molecular Oncology Unit (Lyon, France) (n-74), and in the Cancer Research Unit (Newcastle, United Kingdom) (n-47). In addition 34 NB cell lines were included in the analysis as well as leukocyte DNA from normal individuals and two samples from patients with a distal $2 \mathrm{p} 24$ deletion or duplication, respectively. Tumor DNA was extracted using either the Easy DNA kit following the instructions of the manufacturer (Invitrogen, Carlsbad, CA), a standard proteinase K/SDS procedure using a Phase-lock tube (Eppendorf, Hamburg, Germany) or the Nucleon method (Scotlab, Paisley, Scotland). Leukocyte DNA was extracted from peripheral blood samples using the Blood and Cell Culture DNA mini kit following the instructions of the manufacturer (Qiagen, Hilden, Germany). All DNA samples were stored at $-20^{\circ} \mathrm{C}$.

The MYCN copy number of all tumor samples and cell lines was previously determined using FISH and/or SB. Q-PCR quantifications were performed on 20 tumor DNA samples from Ghent and 74 from France, in total 63 non-MNA samples and 31 MNA samples. To increase the number of primary tumors in the different amplification classes for the survival analysis, 47 additional samples from the UK were analyzed ( 3 of the 47 cases were nonMNA). In addition, 34 NB cell lines were examined.

\section{Real-Time Quantitative PCR Methodology \\ Detection Chemistries and Normalization}

Copy numbers for $M Y C N$ were determined using both TaqMan and SYBR Green I detection chemistry, whereas $D D X 1$ and $N A G$ copy numbers were only determined by the generic dsDNA binding dye SYBR Green I. To account for possible variation related to DNA input amounts or the presence of PCR inhibitors, two reference genes $B C M A$ and $S D C 4$ were simultaneously quantified in separate tubes for each tumor sample.

\section{Quantification}

Quantification was performed using both the standard curve method and the comparative $C_{T}$ method. Standard curves were constructed in each PCR run and the copy numbers of the genes in each sample were interpolated using these standard curves. For the test genes MYCN, DDX1 and NAG, serial dilutions of NB cell line LA-N-1 DNA was 
used as template for the standard curve. For the reference genes, a standard curve was constructed using normal human genomic DNA (Roche Applied Science, Basel, Switzerland). Because these two different DNA samples were used for the construction of the standard curves for either the test and reference genes, the copy numbers of all genes were normalized against a calibrator DNA sample with a disomic copy number of all genes (normal human DNA, Roche).

After normalization to the calibrator, the haploid copy number of MYCN, DDX1 and NAG was calculated by dividing these normalized values by the geometric mean copy number of the two reference genes (BCMA and SDC4).

Instead of interpolating unknown samples from a standard curve, it is also possible to calculate the haploid copy number solely based on the observed $\mathrm{C}_{\mathrm{T}}$ values:

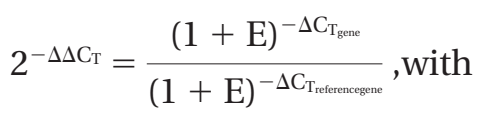

$\mathrm{E}$

$=$ efficiency of the PCR reaction (set at default value 0.95 ),

$\Delta \mathrm{C}_{\mathrm{T}_{\text {gene }}} \quad=$ difference in threshold cycle value between test sample and calibrator sample for the gene under investigation (test gene) and

$\Delta \mathrm{C}_{\mathrm{T}_{\text {referencegene }}}=$ difference in threshold cycle value between test sample and calibrator samle for reference gene.

\section{PCR Conditions}

All primers and probes were designed with Primer Express 1.0 software (Applied Biosystems Foster City, CA) using default TaqMan parameters, with modified minimum amplicon length requirements (85 bp). An additional requirement consisted of a maximum GC content of $40 \%$ for the five last $3{ }^{\prime}$ end nucleotides. The sequence of the primers for the target genes are MYCN F 5' CGCAAAAGCCACCTCTCATTA 3' and MYCN R 5' TCCAGCAGATGCCACATAAGG 3', DDX1 F 5' CCCAACTGATATCCAGGCTGAA 3' and DDX1 R 5' AGTGTGTCCCCAGCTACCAATC 3', NAG F 5' AACATGGACTCGAGAAACCAATTT 3 ' and NAG R 5' TTACTCACTTCCGGCCAGTGT 3'. The primer sequences for the reference genes are BCMA F 5' CGACTCTGACCATTGCTTTCC 3' and BCMA R 5' AAGCAGCTGGCAGGCTCTT 3', SDC4 F 5' CAGGGTCTGGGAGCCAAGT 3' and SDC4 R 5' GCACAGTGCTGGACATTGACA 3'. The sequences of the TaqMan probes are MYCN TET-TTCTGTAAATACCATTGACACATCCGCCTTTTGT-TAMRA, BCMA TET-CAACCATTCTTGTCACCACGAAAACGAATAMRA and SDC4 FAM-CCCACCGAACCCAAGAAACTAGAGGAGAAT-TAMRA.
PCR reactions were performed on an ABI Prism 7700 Sequence Detection System and an ABI Prism 5700 SDS (Applied Biosystems), which yielded similar results (data not shown). Amplification mixtures $(25 \mu \mathrm{l})$ for $M Y C N, D D X 1, N A G, B C M A$ and $S D C 4$ quantification contained template DNA, $1 \times$ SYBR Green I Master Mix buffer (Applied Biosystems) ( $3 \mathrm{~mm} \mathrm{MgCl}_{2}$ ) and $300 \mathrm{~nm}$ of each primer. Amplification mixtures $(25 \mu \mathrm{l})$ for $M Y C N, B C M A$ and SDC4 copy number determination with the TaqMan chemistry consisted of template DNA, $1 \times$ TaqMan buffer A (Applied Biosystems), 500 nм of each primer, $200 \mathrm{~nm}$ of the probe, $1.25 \mathrm{U}$ AmpliTaqGold DNA polymerase (Applied Biosystems), $200 \mu \mathrm{M}$ of each dNTP and $5 \mathrm{~mm} \mathrm{MgCl}_{2}$; or $1 \times$ TaqMan Universal PCR Master Mix (Applied Biosystems), $500 \mathrm{~nm}$ of each primer and $200 \mathrm{~nm}$ of the probe. The cycling conditions comprised 10 minutes polymerase activation at $95^{\circ} \mathrm{C}, 40$ cycles at $95^{\circ} \mathrm{C}$ for 15 seconds and $60^{\circ} \mathrm{C}$ for 1 minute.

Each test gene assay included: 1) a standard curve of five serial 10-fold dilution points of LA-N-1 DNA (ranging from $100 \mathrm{ng}$ to $10 \mathrm{pg}$ ) (in duplicate), 2) a no-template control (in duplicate), 3) $10 \mathrm{ng}$ of calibrator human genomic DNA (Roche) (quadruplicated), and 4) approximately 10 ng of tumor DNA (in duplicate).

Each reference gene assay included: 1) a standard curve of four serial 10-fold dilution points of human genomic DNA (Roche) (ranging from $200 \mathrm{ng}$ to 0.2 ng) (in duplicate), 2) a no-template control (in duplicate), 3) $10 \mathrm{ng}$ of calibrator human genomic DNA (Roche) (quadruplicated), and 4) about $10 \mathrm{ng}$ of tumor DNA (in duplicate). For all duplicated tubes a coefficient of variation (CV) was determined on calculated copy numbers. Samples with a CV higher than $20 \%$ were re-tested.

\section{Primer Concentration Optimization}

A 3-by-3-primer matrix (combinations of 100, 300 and $900 \mathrm{~nm}$ of each forward and reverse primer) was analyzed to determine the optimal concentrations of both forward and reverse primer. The combination of primer concentrations that resulted in the lowest $\mathrm{C}_{\mathrm{T}}$-value (Threshold Cycle), the highest fluorescent signal $\left(\Delta \mathrm{R}_{\mathrm{n}}\right)$ and lack of primer dimer formation or nonspecific amplification was chosen as optimal pair.

\section{Melting Curve Analysis of SYBR Green I Assays}

For each PCR run with SYBR Green I detection, a melting curve analysis was performed to guarantee the specificity in each reaction tube (absence of primer dimers and other nonspecific products). The SDS software supplied with the ABI 5700 allows automatic melting curve analysis for all tested sam- 
ples in a given run. On the ABI 7700, an additional run must be performed after PCR amplification, where SYBR Green I fluorescence of generated products is continuously monitored throughout temperature ramp from $65^{\circ} \mathrm{C}$ to $90^{\circ} \mathrm{C}$ for $14 \mathrm{~min}$ utes. After data recording, a Sequence Detection Software (version 1.7. Applied Biosystems) multicomponent file is exported and imported in a beta version of Dissociation Curves (Applied Biosystems) to display the melting curves and first derivative melting peaks.

FISH Analysis of MYCN, DDX1, NAG, and SDC4 and SB Analysis of $M Y C N$

FISH was performed using the LSI $N$-myc SpectrumOrange probe (Vysis, Downers Grove, IL) or the MYCN digoxigenin labeled probe (Oncor Appligene, Gaithersburg, MD) for the MYCN gene and selected BAC or PAC clones for DDX1 (RP11422A6), NAG (RP11-516B14), and SDC4 (RP3453C12). Labeling and FISH was performed as described (15). SB was performed following described protocols (16-18). The MYCN status in $42 \mathrm{NB}$ tumor samples was determined with both FISH and SB, 79 NB tumor samples were examined with SB only, and $20 \mathrm{NB}$ tumor samples and all NB cell lines were examined with FISH only.

\section{Statistical Analysis}

All data were stored and processed in an Excel sheet. Statistical tests were performed using SPSS 10.0. Overall survival was evaluated from the date of diagnosis to the date of last follow-up or until death occurred. Event free survival was evaluated from the date of diagnosis to the date of last follow up or until relapse or death occurred. Survival analysis was performed using Kaplan Meier graphs and logrank statistics to evaluate the significance of the differences.

\section{RESULTS}

\section{Choice and Evaluation of Reference Genes}

Based on comparative genomic hybridization results of 204 primary NB tumors (19), two genes (BCMA and SDC4) were selected as reference genes. $B C M A$ and SDC4 are located in chromosomal regions that rarely show genetic abnormalities in NB (16p13 and 20q13, respectively). To assess the validity of the genes as appropriate reference genes, we determined the copy number ratio $B C M A / S D C 4$ in 24 normal leukocyte DNA samples and $175 \mathrm{NB}$ tumors and cell lines. The observed ratio measured for normal (1.06 $\pm 0.21 \mathrm{SD})$ and tumor DNA (1.02 \pm $0.41 \mathrm{SD})$ were similar $(P=0.457 \gg 0.05)$ and both significantly equal to $1(P=0.190 \gg 0.05$ and $P=$
$0.552 \gg 0.05$ ), thus confirming their validity as appropriate disomic reference genes in NB.

\section{Comparison of SYBR Green I and TaqMan Chemistries}

For all samples, MYCN copy numbers were determined using SYBR Green I and TaqMan chemistries. A paired sample $t$ test showed that the obtained results by both chemistries were significantly similar $(P=0.872 \gg 0.05)$. In addition, the Spearman correlation coefficient of $0.963(P=1 \mathrm{E}-6 \ll 0.01)$ demonstrated the equivalence of both chemistries. The additional specificity associated with the use of a TaqMan probe compared with the generic DNA binding dye SYBR Green I is compensated by melting curve analysis of the generated PCR products (20). Reactions for all genes and samples were shown to have only one melting peak, which guarantees specific amplification and accurate quantification.

\section{Comparison of Quantification Methods}

Two methods for determination of the haploid copy number (i.e., the MYCN copy number normalized to the disomic copy number of the reference genes) from the raw data of a real-time Q-PCR reaction are available. The relative kinetic method is based on interpolated data from a standard curve, whereas the comparative $\mathrm{C}_{\mathrm{T}}$ method transforms a difference in $\mathrm{C}_{\mathrm{T}}$ values (between the test sample and the calibrator sample) into a copy number ratio.

The relative kinetic method takes the actual efficiency of the reaction into account. For construction of the standard curves of the target genes MYCN, DDX1 and NAG, serial dilutions of NB cell line LA-N-1 DNA were used. Preliminary Q-PCR analysis showed that LA-N-1 contained multiple copies of MYCN, DDX1 and NAG.

The comparative $\mathrm{C}_{\mathrm{T}}$ method is less laborious, as no standard curves are required. We used 0.95 as default amplification efficiency in our comparative $\mathrm{C}_{\mathrm{T}}$ quantification. For all 175 samples the haploid copy number of MYCN, DDX1 and NAG was calculated using both methods. Measurements obtained with both methods were virtually identical (Spearman correlation coefficient $=0.992, P=1 \mathrm{E}-6$ ).

\section{Evaluation of Q-PCR Accuracy}

The accuracy of the assay was tested on DNA from two patients with a constitutional distal $2 p$ deletion and duplication, respectively. The sample with $2 p$ deletion showed a haploid copy number of approximately 0.5 and the sample with $2 p$ duplication showed a haploid copy number of approximately 1.5 (Fig. 1). In addition, the haploid MYCN copy number was measured in 20 normal leukocyte 


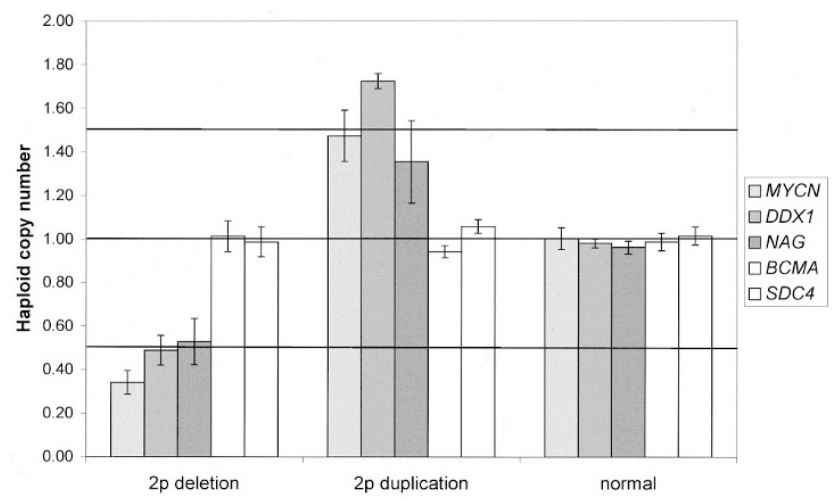

FIGURE 1. Haploid copy numbers ( $\pm \mathrm{SD}$ ) for $M Y C N, D D X 1, N A G$, $B C M A$, and SDC4 in a normal leukocyte DNA sample and DNA samples with $2 \mathrm{p}$ deletion and $2 \mathrm{p}$ duplication, respectively.

samples $(1.14 \pm 0.12 \mathrm{SD})$. These data indicate that single copy changes could be detected. This finding is further supported by Q-PCR and FISH measurements in cell lines SJNB-1 and LA-N-6. The haploid MYCN copy number for SJNB-1 was 1.55. FISH showed six copies for $M Y C N$ and four copies for $S D C 4$, i.e., a 1.5 haploid copy number for MYCN. Similar numbers were found for LA-N-6: 1.63 measured by Q-PCR and 1.5 (3 MYCN/2 SDC4) by FISH.

\section{Comparison of Real-Time Q-PCR and FISH for Assessment of MNA}

FISH and/or SB data for the MYCN status of 175 samples were compared with the Q-PCR results (Table 1). Samples were considered as MNA when the haploid MYCN copy number was higher than 4 (21) (Fig. 2). Results were concordant in 173/175 $(98.9 \%)$ samples. In one MNA tumor (assessed by FISH), Q-PCR measured a MYCN haploid copy number of only 3.00. Re-evaluation of the FISH slide showed, however, that only 5\% MNA cells were present. In NB cell line GI-C-IN, the haploid MYCN copy number was 4.17 as determined by PCR whereas FISH showed a heterogeneous population of cells with a varying $M Y C N$ haploid copy number (1 to 2 ).

TABLE 1. Comparison of Q-PCR Results with FISH or Southern Blot Analysis Results for MYCN Gene Copy Number

\begin{tabular}{lccc}
\hline & \multicolumn{2}{c}{ FISH/SB results } & Total \\
\cline { 2 - 3 } & No MNA & MNA & \\
\hline Q-PCR results & 75 & 1 & 76 \\
No MNA & 1 & 98 & 99 \\
MNA & 76 & 99 & 175 \\
Total & 76 & 99 \\
\hline
\end{tabular}

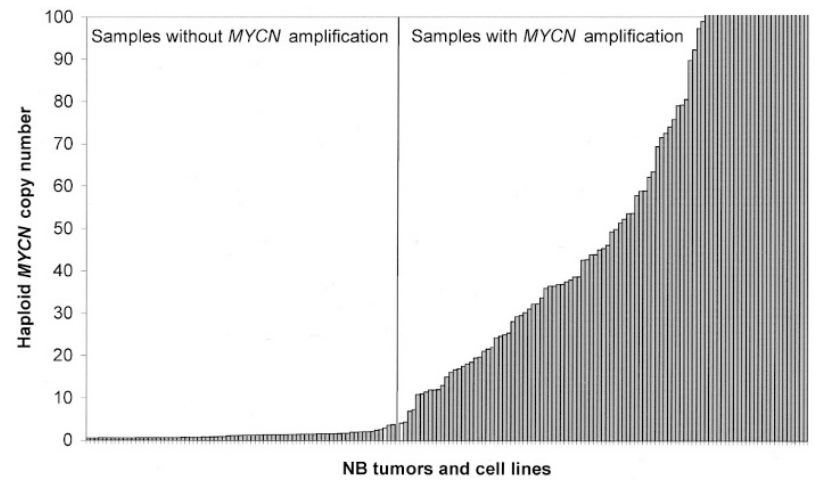

FIGURE 2. Haploid $M Y C N$ copy number of 175 NB samples; the vertical line indicates the cut-off level of four haploid copies. The white bars indicate the two samples with discrepant results (see text).

Minimal Amount of DNA Required for Testing and Effect of Presence of Normal Cells in a Tumor Sample

Determination of MYCN amplification was tested on different amounts of starting material $(0.1,1$, and 10 ng DNA). DNA concentrations were fluorometrically measured using pico-green (Molecular probes) on a TD-360 Mini-Fluorometer (Turner Designs). These Q-PCR tests were performed on two different DNA samples: one with a normal MYCN copy number and one with MNA. It was found that MNA could be detected using as little as $0.1 \mathrm{ng}$ of starting material (Table 2). Coefficient of variation (CV) values below $20 \%$ demonstrate a very good reproducibility.

In addition, we addressed the possibility of MNA detection in tumors with low tumor cell percentage. A normal DNA sample from a healthy individual was serially diluted in a cell line with MNA (CHP902R). MNA could be easily detected in samples with only $20 \%$ tumor cells having a haploid MYCN copy number of 40 (Fig. 3). This finding was supported by a correct classification by Q-PCR of two MNA tumors, which contained only 5 to $10 \%$ MNA tumor cells as assessed by FISH. In these cases, the haploid MYCN copy number determined by Q-PCR was 6.95 and 13.05.

TABLE 2. Calculated Haploid Copy Numbers (CV Based on Two Repeated Experiments) of a Normal Leukocyte DNA Sample (A) and an NB Cell Line with MNA (CHP902R) (B), Using Different Amounts of DNA Template

\begin{tabular}{lccc}
\hline \multicolumn{1}{c}{ (A) Control } & $M Y C N$ & $D D X 1$ & $N A G$ \\
\hline $0.1 \mathrm{ng}$ & $1.11(0.04)$ & $1.19(0.10)$ & $0.98(0.20)$ \\
$1 \mathrm{ng}$ & $0.98(0.18)$ & $1.20(0.18)$ & $0.97(0.10)$ \\
$10 \mathrm{ng}$ & $1.11(0.15)$ & $1.02(0.02)$ & $0.95(0.18)$ \\
\hline (B) CHP902R & $M Y C N$ & $D D X 1$ & $N A G$ \\
\hline $0.1 \mathrm{ng}$ & $37.03(0.10)$ & $25.75(0.18)$ & $30.66(0.11)$ \\
$1 \mathrm{ng}$ & $43.01(0.05)$ & $31.32(0.05)$ & $32.55(0.01)$ \\
$10 \mathrm{ng}$ & $40.99(0.02)$ & $27.65(0.05)$ & $24.84(0.06)$ \\
\hline
\end{tabular}




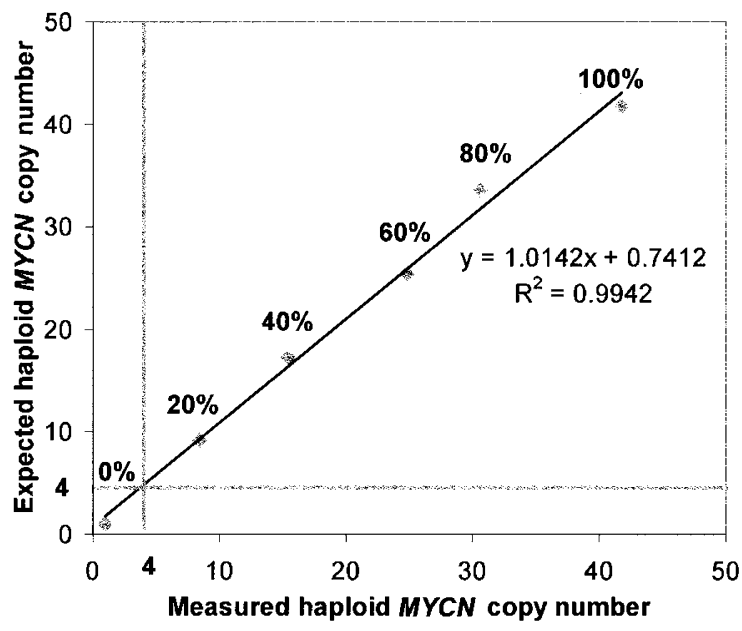

FIGURE 3. Measured and expected $M Y C N$ copy number in samples with diluted MNA NB cell line DNA.

\section{$D D X 1$ and NAG Copy Number Evaluation}

A similar Q-PCR assay strategy was applied for the determination of the amplification status of $D D X 1$ and NAG. DDX1 and NAG copy numbers were accurately determined for the two samples with constitutional $2 \mathrm{p}$ deletion and $2 \mathrm{p}$ duplication (Fig. 1).

In $50 \%(49 / 98)$ of the $M Y C N$ amplified samples DDX1 co-amplification was found (39/75 tumors and 10/23 cell lines). Thirty-four percent (33/98) of the MYCN amplified samples showed $N A G$ amplification (26/75 tumors and 7/23 cell lines). Sixtyseven percent (33/49) of the DDX1 amplified samples are also amplified for NAG (26/39 tumors and $7 / 10$ cell lines). According to the extent of the amplicon, DDX1 only or DDX1 and NAG were coamplified in $M Y C N$ amplified samples. DDX1 and $N A G$ were not amplified in samples without MNA whereas $N A G$ was always co-amplified in samples with $D D X 1$ amplification, in keeping with the varying size of the amplicon and the relative position of the three genes.

\section{Correlation with Clinical Outcome and Co- Amplification of $D D X 1$ and/or NAG}

Figure 4 shows the Kaplan Meier plots for comparison of the survival and event free survival for the different amplification classes (no amplification, MYCN amplification, $M Y C N$ and $D D X 1$ amplification, and MYCN, DDX1 and NAG amplification). Using log-rank statistics no significant difference was found for overall survival and event free survival, whether the patients had an MNA tumor with or without $D D X 1$ and/or NAG amplification.

\section{DISCUSSION}

We have designed and evaluated a Q-PCR assay for measuring $M Y C N$ gene copy number in NB tu-
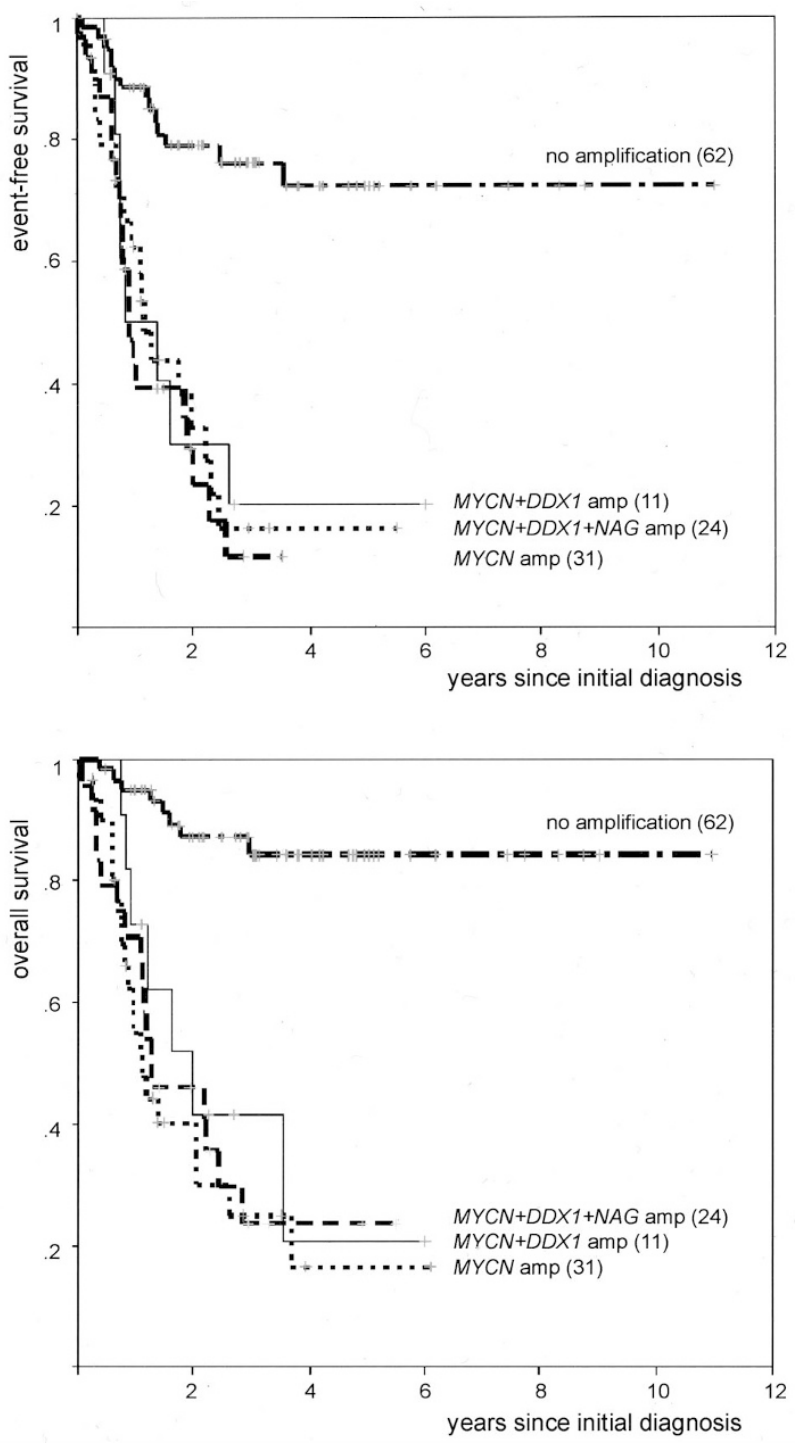

FIGURE 4. Kaplan Meier event free survival (top) and overall survival (bottom) analysis for the different amplification classes.

mor samples. This method can be used as an alternative for $\mathrm{SB}$, which is time-consuming and requires large amounts of fresh or frozen tumor cells, and as a second independent method in parallel with FISH analysis. The recently developed Q-PCR assay allows sensitive fluorescence detection, exponential phase analysis and avoids risk of carry-over contamination (closed tube system). Q-PCR is fast, cheaper and avoids the use of hazardous radioisotopes. However, most importantly, Q-PCR allows the analysis on very small amounts of DNA and offers a wide dynamic range of quantification resulting in accurate values for samples highly differing in their copy number $(22,23)$. Moreover, using the described protocol for MNA assessment, 12 tumor samples can be processed in one 96 well reaction plate in 3.15 hours, including 1 hour hands-on time. 
A number of aspects were critical in the design of a Q-PCR assay for measurement of MYCN, DDX1 and $N A G$ copy numbers. For normalization of the data, two instead of a random single reference gene (24) were chosen. Both genes were selected from chromosomal regions for which copy number changes have been rarely observed (19). The use of two reference genes further increases the reliability of the assay because occasional copy number changes for one gene can be verified by the second reference gene and vice versa. The validity and reliability of both genes as reference genes was demonstrated by measurement of the copy number ratio in normal leukocyte DNA samples and NB tumor and cell lines.

Another important aspect in the Q-PCR assay is the inclusion of an MNA NB cell line for the construction of a standard curve. This approach broadens the dynamic range of the measurements up to 4 log units, compared with the use of disomic copy number DNA (24). Using standard curves the haploid copy number of the target gene can be calculated through interpolation of the raw data. We also evaluated the comparative $\mathrm{C}_{\mathrm{T}}$ method for calculating the gene copy number. This method uses the difference in $\mathrm{C}_{\mathrm{T}}$ values between test and calibrator sample and does not require the construction of standard curves. Our data show that both methods provide identical results. In contrast with other publications $(24,25)$, the accuracy of the present assay was illustrated by detection of single gene copy number changes in two patients with a constitutional $2 p$ deletion or $2 p$ duplication. Real-time Q-PCR results for $173 / 175 \mathrm{NB}$ samples were concordant with FISH and SB data, thus illustrating the value of Q-PCR as a reliable and fast method to determine MNA. One apparent discrepancy could be explained by the low tumor cell percentage (5\%). In NB cell line GI-CI-N, the haploid MYCN copy number was 4.17 as determined by PCR, whereas FISH showed four to six signals for MYCN and three signals for SDC4 (haploid copy number 1 to 2). This difference might be explained by the $M Y C N$ copy number heterogeneity in the cell line and should be investigated in further detail.

The sensitivity of the assay for detection of MNA in the presence of normal cells was determined by a dilution experiment with MNA and normal cells. MNA was still detectable when as little as $20 \%$ tumor cells with a haploid MYCN copy number of \pm 40 were present. In two tumors, only 5 to $10 \%$ estimated MNA tumor cells were still classified as MNA by Q-PCR. In addition to tumor cell percentage, the detection limit for MNA depends on the actual MYCN copy number and the heterogeneity of the tumor cells.

By default we used 10 ng of DNA in each tube, but it was shown that even 100 pg of DNA is sufficient for a reproducible and accurate Q-PCR amplification, which is equivalent to the analysis of approximately 17 diploid cells. This offers the possibility to analyze small amounts of tumor material, e.g., small needle biopsies. In addition, the requirement of only minute amounts of tumor cells offers the possibility to do multiple sampling (e.g., on tumors with different macroscopic appearance), without hampering further pathology investigations.

Up till now, many diagnostic applications of realtime quantitative PCR make use of TaqMan probes for quantification, which adds additional specificity to the reaction. More recently a generic DNA binding dye (SYBR Green I) has found its way in many real-time PCR applications. Using this dye, the specificity of the reaction can be determined by analysis of the melting temperature of the PCR amplicons by generating a so-called melting curve and its first derivative peak (20). In this study Q-PCR results obtained by both chemistries were concordant, thus indicating that SYBR Green I is a valuable alternative for the use of more expensive sequence specific probes. Additionally it has been demonstrated that reliable copy numbers can be measured with the comparative $\mathrm{C}_{\mathrm{T}}$ method.

In the last part of this study, the gene copy number of DDX1 and NAG was also evaluated. Both genes are known to be co-amplified with $M Y C N$ in a subset of NB tumors. The possible additional adverse prognostic effect of both genes has remained unclear so far $(12,13,16,26)$, and was tested on a large set of tumor samples in this study. Amplification of DDX1 and NAG was found in half and onethird of nearly 100 MNA NB samples, respectively. These findings are in keeping with the literature data with the exception of a higher $N A G$ coamplification percentage in a study by Wimmer and colleagues (14), which might be explained by the small number of tumors tested in their study. Kaplan Meier survival analysis demonstrated that $D D X 1$ or $N A G$ co-amplification did not result in additional unfavorable prognostic effect.

In conclusion, the present assay offers a powerful tool for $M Y C N$ gene copy number analysis in NB tumors and can be recommended as a second independent technique in addition to FISH analysis. In this study, Q-PCR was performed on fresh $\mathrm{NB}$ samples. We are currently evaluating this Q-PCR approach of MYCN copy number measurements on paraffin embedded tumor material.

Acknowledgments: We thank R. Godbout and K. Wimmer for providing us with partial intron sequences of, respectively, DDX1 and NAG. R. Pauwels (Ghent, Belgium) is gratefully acknowledged for the use of the ABI7700, and L. Messiaen (Ghent, Belgium) for providing us with normal control leuko- 
cyte DNA samples. R. Versteeg (Amsterdam, The Netherlands), P. Ambros (Vienna, Austria), P. Reynolds (Los Angeles), G. Brodeur (Philadelphia), T. Look (Boston), and S. Cohn (Chicago) are gratefully acknowledged for providing us with NB cell lines. We thank G. De Vos, P. Degraeve (Ghent, Belgium), and M. Van Dongen (Rotterdam, The Netherlands) for culturing of the cells and cell lines.

\section{REFERENCES}

1. Maris JM, Matthay KK. Molecular biology of neuroblastoma. J Clin Oncol 1999;17:2264-79.

2. Brodeur GM, Pritchard J, Berthold F, et al. Revisions of the international criteria for neuroblastoma diagnosis, staging, and response to treatment. J Clin Oncol 1993;11:1466-77.

3. Rubie H, Hartmann O, Michon J, et al. $N$-Myc gene amplification is a major prognostic factor in localized neuroblastoma: results of the French NBL 90 study. Neuroblastoma Study Group of the Societe Francaise d'Oncologie Pediatrique J Clin Oncol 1997;15:1171-82.

4. Bown N, Cotterill S, Lastowska M, et al. Gain of chromosome arm 17q and adverse outcome in patients with neuroblastoma. N Engl J Med 1999;340:1954-61.

5. Shapiro DN, Valentine MB, Rowe ST, et al. Detection of $N$-myc gene amplification by fluorescence in situ hybridization. Diagnostic utility for neuroblastoma. Am J Pathol 1993;142:1339-46.

6. Strehl S, Ambros PF. Fluorescence in situ hybridization combined with immunohistochemistry for highly sensitive detection of chromosome 1 aberrations in neuroblastoma. $\mathrm{Cy}$ togenet Cell Genet 1993;63:24-8.

7. Oude Luttikhuis ME, Iyer VK, Dyer S, Ramani P, McConville $\mathrm{CM}$. Detection of MYCN amplification in neuroblastoma using competitive PCR quantitation. Lab Invest 2000;80:271-3.

8. Huddart SN, Mann JR, McGukin AG, Corbett R. MYCN amplification by differential PCR. Pediatr Hematol Oncol 1993;10:31-4.

9. Higuchi R, Fockler C, Dollinger G, Watson R. Kinetic PCR. analysis: real-time monitoring of DNA amplification reactions. Biotechnology (NY) 1993;11:1026-30.

10. Heid CA, Stevens J, Livak KJ, Williams PM. Real time quantitative PCR. Genome Res 1996;6:986-94.

11. Godbout R, Packer M, Bie W. Overexpression of a DEAD box protein (DDX1) in neuroblastoma and retinoblastoma cell lines. J Biol Chem 1998;273:21161-8.

12. Manohar CF, Salwen HR, Brodeur GM, Cohn SL. Coamplification and concomitant high levels of expression of a DEAD box gene with MYCN in human neuroblastoma. Genes Chromosom Cancer 1995;14:196-203.

13. Squire JA, Thorner PS, Weitzman S, et al. Co-amplification of MYCN and a DEAD box gene (DDX1) in primary neuroblastoma. Oncogene 1995;10:1417-22.
14. Wimmer K, Zhu XX, Lamb BJ, et al. Co-amplification of a novel gene, NAG, with the $N$-myc gene in neuroblastoma. Oncogene 1999;18:233-8.

15. Van Roy N, Laureys G, Cheng NC, et al. 1;17 translocations and other chromosome 17 rearrangements in human primary neuroblastoma tumors and cell lines. Genes Chromosom Cancer 1994;10:103-14.

16. George RE, Kenyon RM, McGuckin AG, Malcolm AJ, Pearson $\mathrm{AD}$, Lunec J. Investigation of co-amplification of the candidate genes ornithine decarboxylase, ribonucleotide reductase, syndecan- 1 and a DEAD box gene, DDX1, with $N$-myc in neuroblastoma. United Kingdom Children's Cancer Study Group. Oncogene 1996;12:1583-7.

17. George RE, Kenyon R, McGuckin AG, et al. Analysis of candidate gene co-amplification with MYCN in neuroblastoma. Eur J Cancer 1997;33:2037-42.

18. Combaret V, Wang Q, Favrot MC, et al. Clinical value of $\mathrm{N}$-myc oncogene amplification in 52 patients with neuroblastoma included in recent therapeutic protocols. Eur J Cancer Clin Oncol 1989;25:1607-12.

19. Vandesompele J, Speleman F, Van Roy N, et al. Multicentre analysis of patterns of DNA gains and losses in 204 neuroblastoma tumors: How many genetic subgroups are there? Med Pediatr Oncol 2001;36:5-10.

20. Ririe KM, Rasmussen RP, Wittwer CT. Product differentiation by analysis of DNA melting curves during the polymerase chain reaction. Anal Biochem 1997;245:154-60.

21. Ambros PF, Ambros IM, for the SIOP Europe Neuroblastoma Pathology, Biology, and Bone Marrow Group. Pathology and biology guidelines for resectable and unresectable neuroblastic tumors and bone marrow guidelines. Med Pediatr Oncol 2001;37:489-91.

22. Bieche I, Olivi M, Champeme MH, Vidaud D, Lidereau R, Vidaud M. Novel approach to quantitative polymerase chain reaction using real-time detection: application to the detection of gene amplification in breast cancer. Int J Cancer 1998;78:661-6.

23. Lie YS, Petropoulos CJ. Advances in quantitative PCR technology: 5' nuclease assays. Curr Opin Biotechnol 1998;9: 43-8.

24. Raggi CC, Bagnoni ML, Tonini GP, et al. Real-time quantitative PCR for the measurement of MYCN amplification in human neuroblastoma with the TaqMan detection system. Clin Chem 1999;45:1918-24.

25. Tajiri T, Tanaka S, Shono K, et al. Quick quantitative analysis of gene dosages associated with prognosis in neuroblastoma. Cancer Lett 2001;166:89-94.

26. George RE, Thomas H, McGuckin AG, et al. The DDX1 gene which is frequently co-amplified with MYCN in primary neuroblastoma is itself tumourigenic. Proc Am Assoc Cancer Res 1998;39:471. 